\title{
Gamma rhythms in the visual cortex: functions and mechanisms
}

\author{
Chuanliang $\operatorname{Han}^{1} \cdot$ Robert Shapley $^{2} \cdot$ Dajun Xing $^{1}$
}

Received: 1 July 2021 / Revised: 9 November 2021 / Accepted: 5 December 2021 / Published online: 22 December 2021

(C) The Author(s) 2021

\begin{abstract}
Gamma-band activity, peaking around $30-100 \mathrm{~Hz}$ in the local field potential's power spectrum, has been found and intensively studied in many brain regions. Although gamma is thought to play a critical role in processing neural information in the brain, its cognitive functions and neural mechanisms remain unclear or debatable. Experimental studies showed that gamma rhythms are stochastic in time and vary with visual stimuli. Recent studies further showed that multiple rhythms coexist in V1 with distinct origins in different species. While all these experimental facts are a challenge for understanding the functions of gamma in the visual cortex, there are many signs of progress in computational studies. This review summarizes and discusses studies on gamma in the visual cortex from multiple perspectives and concludes that gamma rhythms are still a mystery. Combining experimental and computational studies seems the best way forward in the future.
\end{abstract}

Keywords Gamma rhythm · Visual cortex · Neural network · Dynamic system · Computational Model

\section{Significance of gamma oscillations}

Gamma rhythms $(30-100 \mathrm{~Hz})$ in the local field potential (LFP) are commonly found in many brain regions (Buzsáki 2009; Wang 2010), including the hippocampus (Bragin et al. 1995; Wang and Buzsáki 1996; Colgin et al. 2009; Belluscio et al. 2012; Fernández-Ruiz et al. 2021), entorhinal cortex (Chrobak and Buzsáki 1998; Quilichini et al. 2010), olfactory bulb (Adrian 1942, 1950; Neville and Haberly 2003), auditory cortex (Lakatos et al. 2005; Fujioka et al. 2009; Vianney-Rodrigues et al. 2011; Gross et al. 2013), parietal cortex (Bouyer et al. 1981; Pesaran et al. 2002; Hawellek et al. 2016), prefrontal cortex (Gregoriou et al. 2009; Benchenane et al. 2011; Colgin 2011; Kim et al. 2016), and visual cortex (Eckhorn et al. 1988; Gray and Singer 1989a; Gray et al. 1989; Frien et al. 1994; Kreiter and Singer 1996; Friedman-Hill et al. 2000;

Dajun Xing

dajun_xing@bnu.edu.cn

1 State Key Laboratory of Cognitive Neuroscience and Learning \& IDG/McGovern Institute for Brain Research, Beijing Normal University, Beijing 100875, China

2 Center for Neural Science, New York University, New York, NY, USA
Maldonado et al. 2000; Hermes et al. 2015). It has been proposed that gamma-band activity may play an essential role in normal cognitive processes (Eckhorn et al. 1988; Gray et al. 1989; Singer and Gray 1995; Draguhn and Buzsáki 2004; Henrie and Shapley 2005; Fries 2005, 2009; Fries et al. 2007; Wang 2010; Buzśaki and Wang 2012) such as learning (Bauer et al. 2007), memory (Pesaran et al. 2002; Kucewicz et al. 2017), and attention (Fries et al. 2001; Jensen et al. 2007; Womelsdorf and Fries 2007; Vinck et al. 2013). Abnormal gamma rhythms are associated with mental disease (Uhlhaas and Singer 2006, 2010, 2012; Gonzalez-Burgos and Lewis 2012; Lewis et al. 2012).

Gamma-band activity was first discovered in the cat olfactory bulb (Adrian 1942, 1950). Then years later the presence of gamma activity in the primary visual cortex, V1, started drawing attention, first in cat (Eckhorn et al. 1988; Gray and Singer 1989b; Gray et al. 1989; Gray and Di Prisco 1997; Samonds and Bonds 2005), then in macaque monkey (Frien et al. 1994; Kreiter and Singer 1996; Friedman-Hill et al. 2000; Maldonado et al. 2000; Lima et al. 2010; Ray and Maunsell 2011; Jia and Kohn 2011; Womelsdorf et al. 2012; Xing et al. 2012b; Roberts et al. 2013; Jia et al. 2013a; Van Kerkoerle et al. 2014; Murty et al. 2018; Peter et al. 2019; Onorato et al. 2020) and in humans (Tallon-Baudry and Bertrand 1999; Canolty 
et al. 2006; Hermes et al. 2015; Michalareas et al. 2016; Kucewicz et al. 2017). Rodents (Cardin et al. 2005, 2009; Saleem et al. 2017; Storchi et al. 2017; Welle and Contreras 2017) and insects (Kirschfeld 1992; Grabowska et al. 2020) also became animal models for understanding the neural mechanisms of gamma-band activity.

In the visual cortex, gamma is observed in the local field potential (LFP) as a narrow-band increase in power in the gamma frequency range (around $30-80 \mathrm{~Hz}$ ) during the presentation of a visual stimulus (Fig. 1A-C) (Gray and Singer 1989a; Castelo-Branco et al. 1998; Fries et al. 2007; Xing et al. 2012a; Jia et al. 2013b). The bell-shaped bump (narrow-band gamma) in the power spectrum is defined as a gamma rhythm. In the LFP, a broad-band power elevation over a wide frequency range around the gamma band (Fig. 1D) (Henrie and Shapley 2005; Yuval-Greenberg et al. 2008; Jia et al. 2011; Ray and Maunsell 2011) is a different component, which behaves similarly to spiking activity (Jia et al. 2011; Ray and Maunsell 2011). In the following sections of this paper, we focus on studies of narrow-band gamma in the visual cortex of cat and monkey, the two animal models in which studies and theories of gamma rhythms were initiated.

\section{The functions of gamma oscillation are controversial}

There have been many speculations about the function of gamma-band activity. For example, gamma synchronization may contribute to solving the 'binding problem' (Singer and Gray 1995; Singer 1999a). The activity of neurons could be grouped together dynamically through synchrony associated with perceptual Gestalt principles (Milner 1974; Engel et al. 1992; von der Malsburg 1994). Engaging in an oscillation could also increase the saliency of neuronal signals (Gray and McCormick 1996) such as the relationship between orientation discrimination performance and gamma band activity (Edden et al. 2009). In $\mathrm{V} 1$, orientation selectivity was modulated by the gamma phase (Womelsdorf et al. 2012) and the changes of orientation preference of neuronal populations in visual cortex occurred if stimuli induced synchronized responses oscillating at gamma band frequencies (Galuske et al. 2019).

The early studies on cats (Eckhorn et al. 1988; Gray and Singer 1989a; Gray et al. 1989; Gray and Di Prisco 1997) provided evidence that global features (such as contour information, or motion information at different visual locations that belonged to the same object) of visual stimuli were associated with robust synchronization of responses in V1. These observations were then taken as support for the binding-by-synchrony (BBS) hypothesis (Milner 1974;
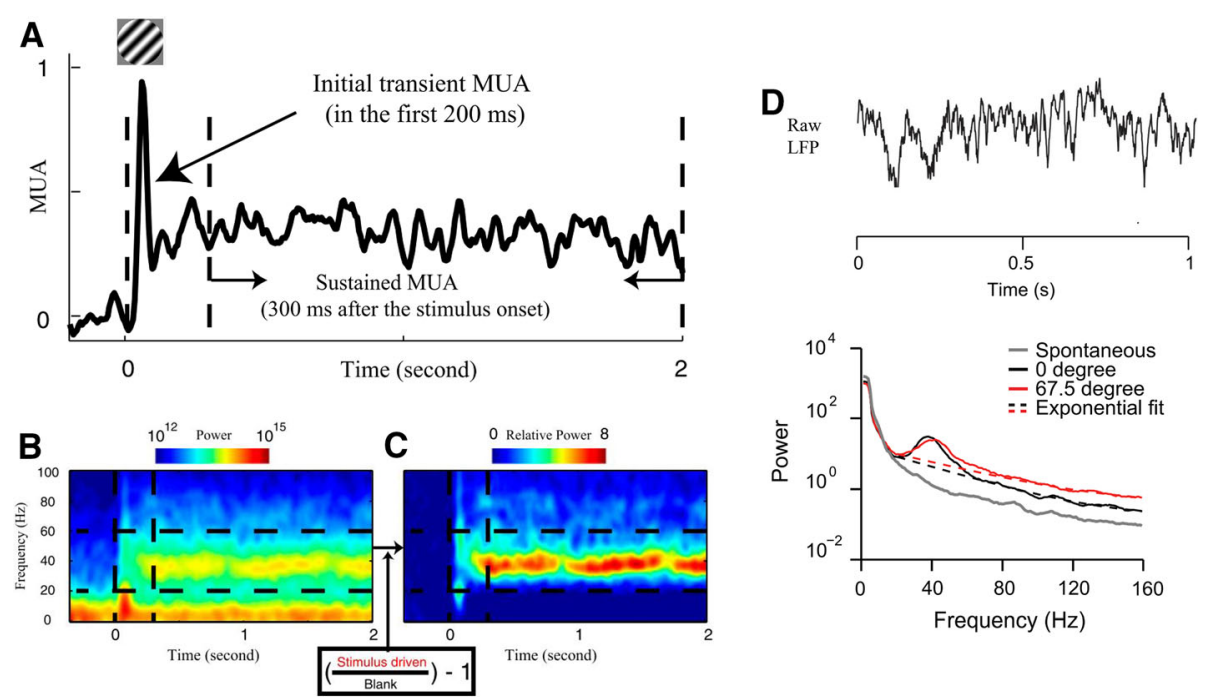

Fig. 1 Stimulus induced narrow-band and broad-band gamma activity. A-C are replotted based on Xing et al. (2012a). A shows the multiunit activity to the visual stimulus (drifting grating) for an example site. The response over the first $0.2 \mathrm{~s}$ after stimulus onset was termed the initial transient response. The response from 0.3 to $2 \mathrm{~s}$ after stimulus onset (time 0) was taken to be the sustained response. B illustrates the color-coded spectrogram (power spectrum vs time) of the Local Field Potential (LFP) to the drifting grating stimulus. The sustained power is induced after stimulus onset (time 0 marked by the first vertical dashed line) in the frequency band between 20 and $60 \mathrm{~Hz}$ (marked by two horizontal dashed lines). $\mathbf{C}$ shows the relative power spectrum which is normalized by power at each frequency during the blank period. D is replotted based on Jia et al. (2011). The top panel of $\mathbf{D}$ shows a single epoch example of the raw LFP and the bottom panel shows the power spectra of LFPs from one site for two orientations (red and black lines) and spontaneous activity (gray lines). The gamma bump around $40 \mathrm{~Hz}$ is defined as the narrow-band component of gamma. The broad-band component of gamma power is estimated by the exponential fit indicated with dashed lines 
Grossberg 1976; von der Malsburg 1994), that is, that binding/integration of information for a visual object might be through synchronization by gamma rhythms in V1 (Singer 1999b, 2009; Uhlhaas and Singer 2006, 2010, 2012). However, the BBS hypothesis has been challenged by the proposal that gamma is an epiphenomenon that is irrelevant for information processing (Shadlen and Movshon 1999). Furthermore, experimental data indicate that the peak frequency in the gamma band varies across visual cortical loci when they are activated by the same visual object (Ray and Maunsell 2010). Although there are some possible explanations of these problems for the BBS hypothesis in a follow-up study (Singer 2021), the controversy about the BBS hypothesis continues.

Several years after the BBS hypothesis was proposed came the 'communication-through-coherence' (CTC) hypothesis (Fries 2005, 2009). The CTC hypothesis proposed that gamma rhythms facilitate neuronal communication between cortical regions by neuronal coherence in the gamma-band and the effectiveness of long-distance communication depends on the relative gamma phase (zero phase) between the spikes and the LFP. Similar to the situation with the BBS hypothesis, the CTC hypothesis was both supported (Womelsdorf et al. 2007; Gregoriou et al. 2009) and opposed (Jia et al. 2013a, Akam and Kullmann 2012). An updated version of the CTC hypothesis (Fries 2015) proposed that entrainment with delay was the mechanism that sets up phase relations subserving CTC. The updated CTC proposed also that the way rhythmic synchronization modulates excitability is not simply by linear filtering of a sinusoidal drive, but with nonlinear models composed of spiking excitatory and inhibitory neurons (Borgers and Kopell 2008; Gielen et al. 2010). At present, more studies support the CTC hypothesis than oppose it, but more experiments and theories are needed to verify it in the future.

Both the BBS and CTC hypotheses implied that gamma activity performs important functions for perception or cognition. However, many studies in recent years do not support the idea that gamma rhythms in the visual cortex have specific visual functions (Singer 2018). Instead, many people think that gamma-band activity is a by-product of neuronal activity in the cortical network (Shadlen and Movshon 1999; Thiele and Stoner 2003; Roelfsema et al. 2004; Henrie and Shapley 2005; Ray and Maunsell 2010; Chariker et al. 2016, 2018) or in some cases gamma may be an artifact (Yuval-Greenberg et al. 2008). The arguments against the idea that synchronized oscillations have a functional role came from empirical studies on response properties of gamma rhythms, including studies of stimulus-dependence, stochastic dynamics, and differences of gamma-band activity in different species.

\section{What visual information does gamma oscillation represent?}

Although gamma has been thought important for visual processing, what is represented by gamma is still unclear. Some previous studies showed that gamma is strongly induced by thin bars or face contours (Fig. 2A) (Gray and Singer 1989b; Singer and Gray 1995; Neuenschwander and Singer 1996; Castelo-Branco et al. 1998; Uhlhaas and Singer 2006, 2010, 2012). Such results suggested that gamma could represent a visual object or the contour of a visible object. But other studies demonstrated that the surfaces of visual stimuli also induce strong gamma-synchronous responses in V1 (Fig. 2B) (Gail et al. 2000; Xing et al. 2010, 2014a; Peter et al. 2019), suggesting that the gamma rhythm could represent surface information (Peter et al. 2019). Whether gamma represents a visual object's contour or its surface is still unclear.

The surface preference of gamma band activity can be modified by surface properties. For instance, gamma peaks in the power spectrum of the LFP were strongly induced by a black surface, but the gamma peak was weak or absent for a white surface in macaque V1 (Xing et al. 2014) (Fig. 2C) or cat V1 (Fig. 2D, unpublished data from Dajun Xing's lab). A similar result could also be seen in the color preference of gamma; gamma prefers red surfaces more than surfaces of other colors (Shirhatti and Ray 2018; Peter et al. 2019). If gamma represents visual surfaces, why there is such a strong luminance/color preference? One possible answer is that it could be simply a consequence of different cone contrast-red versus gray higher in cone contrast than green versus gray, for instance. But the color preference still needs to be confirmed and explored more deeply in future experiments.

The uncertainty about gamma's function for information representation may be due to the fact that we don't understand fully the mechanisms for the stimulus dependence of gamma band rhythms in the visual cortex. Previous studies have shown that gamma depends on different stimulus characteristics, such as luminance/color (Swettenham et al. 2013; Xing et al. 2014; Saleem et al. 2017; Storchi et al. 2017; Shirhatti and Ray 2018; Peter et al. 2019), contrast (Henrie and Shapley 2005; Ray and Maunsell 2010; Jia et al. 2013b), orientation (Siegel and König 2003; Kayser and Ko 2004; Cardin et al. 2005; Berens et al. 2008; Womelsdorf et al. 2012; Han et al. 2020), temporal frequency (Jia et al. 2011; Murty et al. 2018), stimulus size (Bauer et al. 1995; Gieselmann and Thiele 2008; Jia et al. 2011, 2013b; Ray and Maunsell 2011). Furthermore, the complexity of visual stimuli modulates the strength of cortical gamma activity. Gamma band power is reduced when the visual stimulus includes a 
Fig. 2 Gamma oscillation induced by surface or object stimuli. A is replotted based on Castelo-Branco et al. (1998).

The bar stimulus evokes strong oscillatory responses at $49 \mathrm{~Hz}$ in Area 18 of the cat, as indicated by the oscillatory spectrum of spike times in the righthand panel. B is replotted based on Peter et al. (2019). It shows average LFP power spectra for different chromatic conditions. $\mathrm{C}$ is replotted based on Xing et al. (2014). It shows average LFP power spectra to black and white stimuli in layer 4C of V1. D shows unpublished data from Dajun Xing's lab, which shows average LFP power spectra to black and white stimuli in cat V1
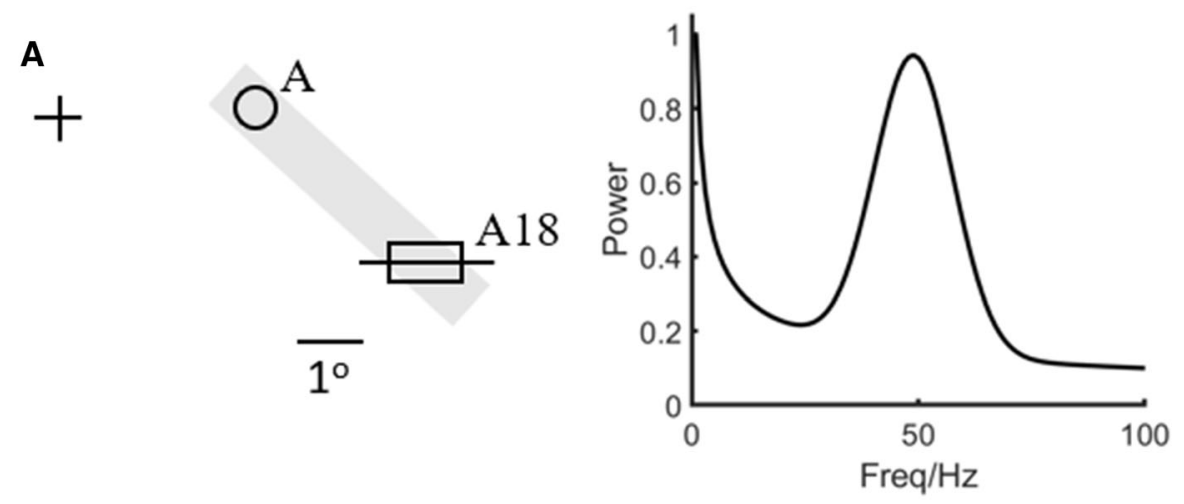

B

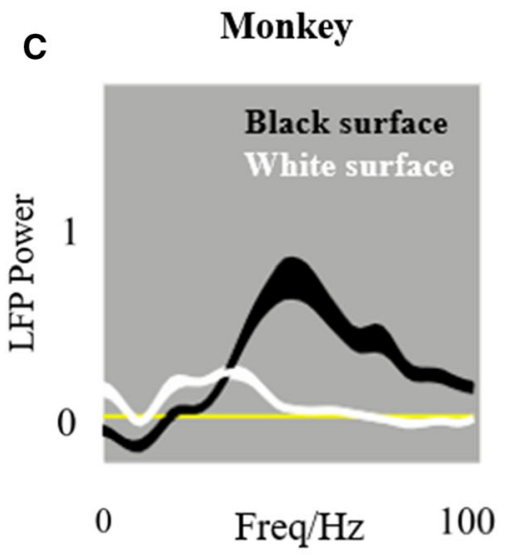

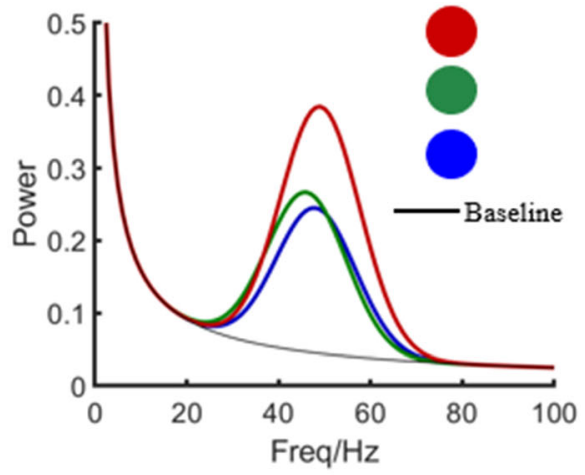

Cat

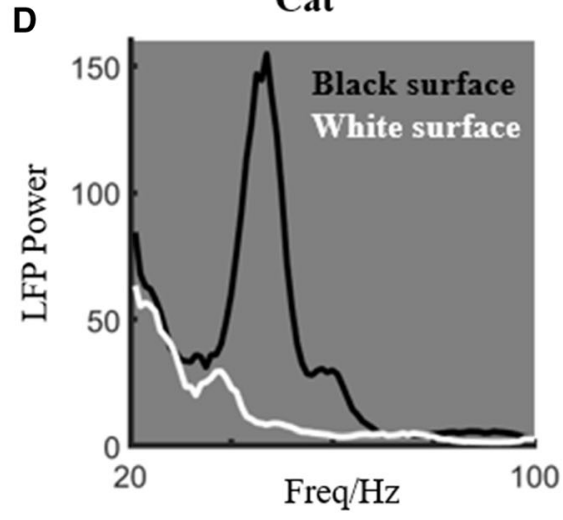

noise masking grating (Zhou et al. 2008; Jia and Kohn 2011; Jia et al. 2013a), and in natural images(Kayser et al. 2003; Kayser and Ko 2004; Hermes et al. 2015), and when the visual stimulus consists of superimposed gratings (Lima et al. 2010; Bartolo et al. 2011; Wang et al. 2021). In the last section of our review, we will discuss progress in understanding the stimulus-dependence of gamma rhythms.

\section{How does gamma band activity help information integration?}

In addition to visual information representation, the relationship between gamma rhythms and visual information integration is also unclear. Spike activity, which carries visual information, has been found to lock to the phase of the gamma rhythm (Schoffelen et al. 2005; Fries et al. 2007; Fries 2015). The strong phase-locking between visually-evoked signals and gamma suggests that different kinds of visual information might be communicated according to their gamma phase. Perhaps phase-locking together with the CTC hypothesis (Fries 2005, 2009, 2015) could explain how different visual signals about visual objects could be integrated in downstream cortical regions. 
However how the visual system coordinates/integrates gamma information in time and phase is not fully understood. Some theoretical work has used oscillator models to understand gamma rhythm (Börgers 2017). A natural consequence of the oscillator theories is the concept of gamma as a clock signal for synchronizing brain information. However, experimental studies found that gamma rhythms in V1 are not oscillatory but instead are stochastic in time (Burns et al. 2010, 2011; Xing et al. 2012a) (Fig. 3, but see Subhash Chandran et al. 2018). There are rapid changes of frequency and only short durations of consistent phase and power elevation (Also see Feingold et al. 2015 for that of beta rhythm in motor cortex and Bastos et al. 2018 for gamma bursting in frontal cortex). The nonoscillatory nature of gamma was shown theoretically to cause more CTC (Saraf and Young 2021) suggesting that instaneous synchronization, instead of sustained oscillation, is more important for information integration (Xing et al. 2012a). Although there are studies (Jia et al. 2013a; Roberts et al. 2013) that showed that gamma's stochastic properties are consistent and coordinated between monkey V1 and V2, we still don't know the exact way that the brain integrates the information carried by gamma in time. We need to rethink how gamma rhythms are generated and how gamma rhythms might aid information processing in the brain.

Besides the randomness of gamma in time, the gamma rhythm is also variable in space. Ray and Maunsell (2010) showed that frequencies of gamma rhythms could be continuously different as a function of location in visual space, even if the different locations belong to the same visual object. The frequency difference for gamma in space is somewhat puzzling; if specific visual information appears in a fixed phase for all different frequencies, then the information carried by these frequencies will appear at different times; if the visual system synchronizes the information at the same time, then the information will appear in different phases for various frequencies.

Most studies on gamma band activity have reported a single gamma frequency bump in monkey V1. Interestingly, two gamma peaks in the frequency spectrum have been previously reported in the visual cortex of humans (Kucewicz et al. 2017), cats (Fig. 4A-C) (Castelo-Branco et al. 1998; Bharmauria et al. 2016; Han et al. 2020; Wang et al. 2021), monkeys (Murty et al. 2018 and Fig. 4D is unpublished data for awake monkey V1 from Dajun Xing's lab) and rats (Oke et al. 2010). The finding of multiple gammas generates more questions about gamma's function. What kind of visual information is carried by two distinct gamma peaks, and what is the relationship between the two gamma peaks? How do downstream cortical regions integrate information carried by multiple gammas? All these questions have not been answered fully.

\section{Do gamma rhythms serve a canonical function?}

If gamma band activity has essential functions in cognition and perception, one should expect that its importance will lead to conservation of brain functions and circuits that generate gamma in different species throughout biological evolution. However, this seems not to be the case; instead, recent studies have shown that gamma band activity in different species' visual cortices are different in response properties and neural origins.

Bastos et al. (2014), studying macaque monkeys, found gamma in V1 (specifically in V1 superficial layers Spaak et al. 2012; Xing et al. 2012b; Roberts et al. 2013; Van Kerkoerle et al. 2014) but not in the LGN and concluded that a visually-induced gamma rhythm is an emergent property in the cortex (Fig. 5A). However, for mice,
Fig. 3 Stochastic generation of gamma in awake and anesthetized states. The figure is replotted based on Xing et al. (2012b). The top panel shows the LFP recorded from an awake monkey after stimulus onset and in the panel below it is its spectrogram. The gray shaded areas of the LFP are gamma-band bursts. Each black bar in the spectrogram marks the peak frequency (y-axis) and the duration of each gammaband burst

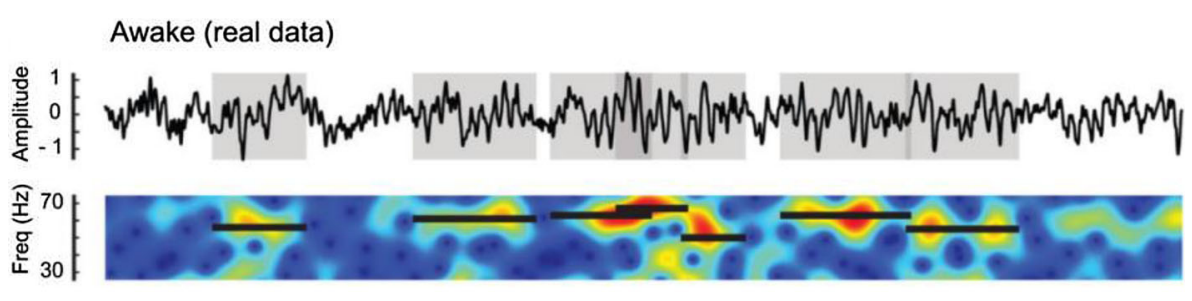

Awake (surrogate data)

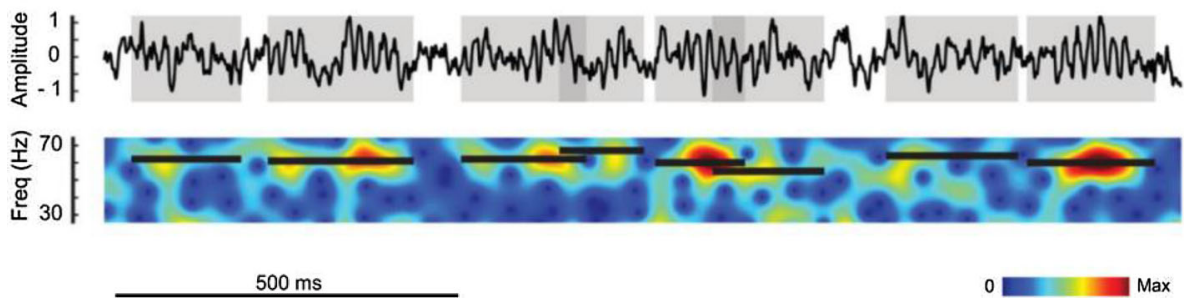



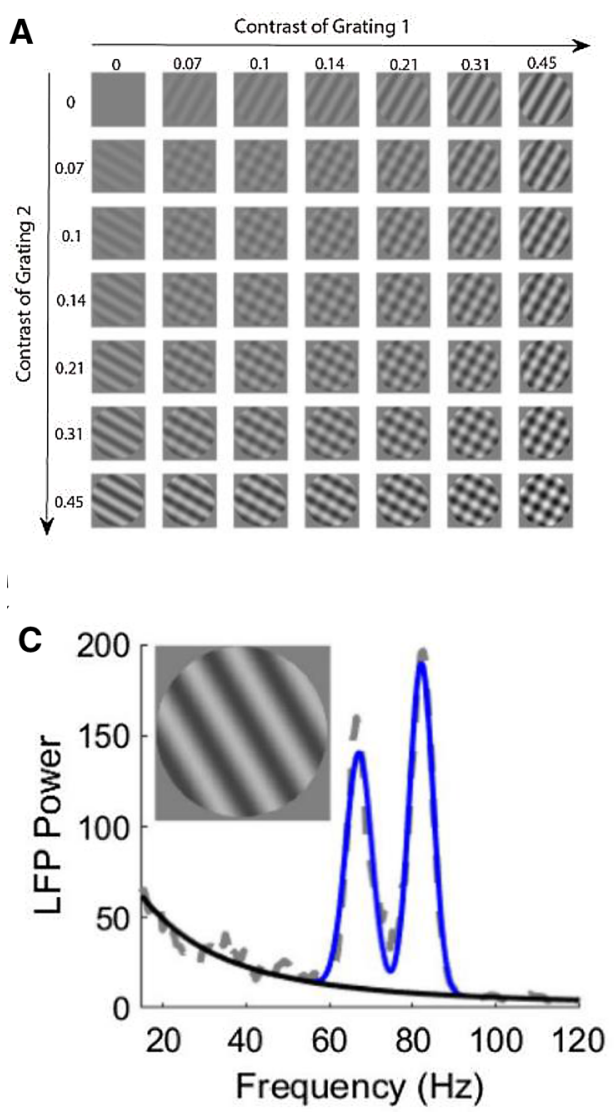

Fig. 4 Experimental evidence for multiple gamma oscillations in the visual cortex. A-C is replotted based on Wang et al. (2021). A and B show the response matrix driven by plaid for an example site. A presents various plaids formed by the linear summation of two orthogonal drift gratings with varying contrast. These stimuli (size, $38^{\circ}$; spatial frequency, 0.05 cycle/deg) were presented for $2 \mathrm{~s}$ with a time-frequency of $2 \mathrm{~Hz}$ and were repeated 10 times. B shows the trial averaged $(n=10)$ LFP power spectrum (gray dots) in response to

studies have shown that gamma is generated in subcortical regions, including LGN (Saleem et al. 2017; Schneider et al. 2021) and retina (Storchi et al. 2017) (Fig. 5B). Studying cat visual cortex, Castelo-Branco et al., (1998) found two narrow-band gamma peaks. Their results indicated that one is generated at the cortical level, and the other one is of subcortical origin. More importantly, the response properties of gamma rhythms are also speciesspecific. In macaque, Xing et al. (2014) found that the peak in the gamma band preferred dark stimuli (Figs. 2D, 5C for macaque data; also see Fig. 2D for unpublished data in cats from Dajun Xing's lab), while in mouse V1, the LFP gamma activity was strongly induced by lightness instead of darkness (Fig. 5D) (Saleem et al. 2017; Storchi et al. 2017). The species differences are not consistent with a canonical role of gamma in cognition and perception.
B

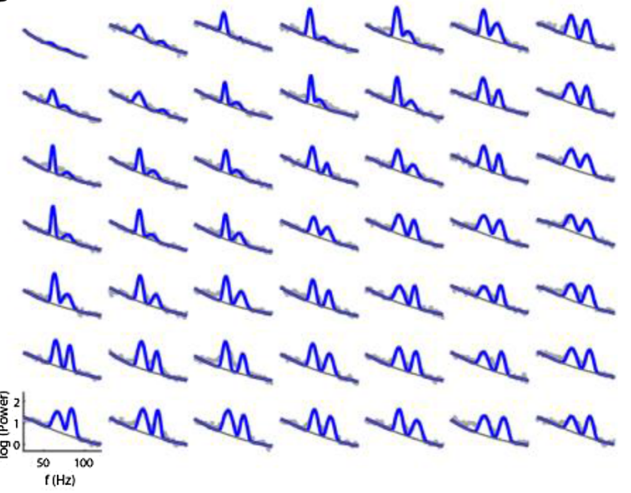

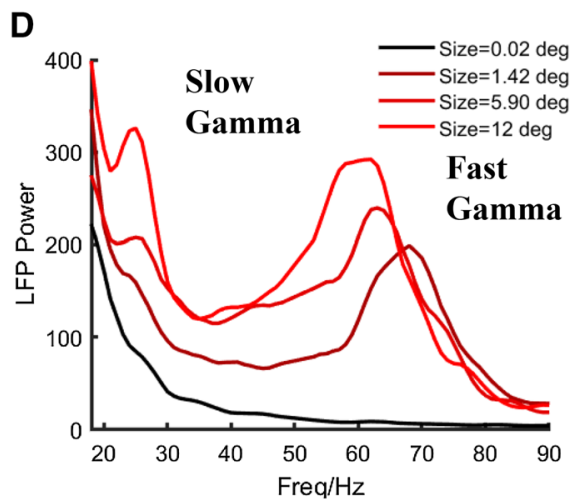

corresponding stimuli in A. Two narrow-band gamma oscillations (blue curve) and the baseline (dark gray line) were estimated through a spectrum fitting procedure. $\mathbf{C}$ shows the LFP power spectra have two distinct narrow-band gamma oscillations induced by a grating in cat V1. D shows the LFP power spectra in awaked macaque V1 that have two distinct narrow-band gamma oscillations induced by gratings with different size, which is the unpublished data from Dajun Xing's lab

\section{Neural mechanism of gamma rhythms revealed by computational models}

We have to admit that we don't fully understand the function, or functions, of gamma rhythms given all the puzzles and controversies reviewed above. This is primarily due to the fact that we also don't know the neural mechanisms for generating and modulating gamma. With advanced experimental techniques (intracellular recordings, optogenetics, etc.), mouse studies have revealed excitatory and inhibitory contributions to gamma (Cardin et al. 2009; Veit et al. 2017). But so far these techniques cannot be fully applied in primate studies. The species difference for gamma properties suggests a need for separate studies on different species. An alternative way to understand gamma at the circuit level for primates is to construct dynamic models of neural networks combining experimental data. 
Fig. 5 Species difference on generation mechanism and functions of gamma rhythms. $\mathbf{A}$ is plotted based on the main result in Bastos et al. (2014) which shows the cortical mechanism of the gamma rhythm. A is plotted based on the main result in Saleem et al. (2017) which shows the subcortical mechanism of the gamma rhythm. $\mathbf{C}$ is replotted based on Xing et al. (2014). It shows the average power spectra (LFP) to black and white stimuli in macaque V1. D is replotted based on Storchi et al. (2017) and Saleem et al. (2017). It shows the average LFP power spectra during uniform light conditions (white) or complete darkness (black)
A Macaque
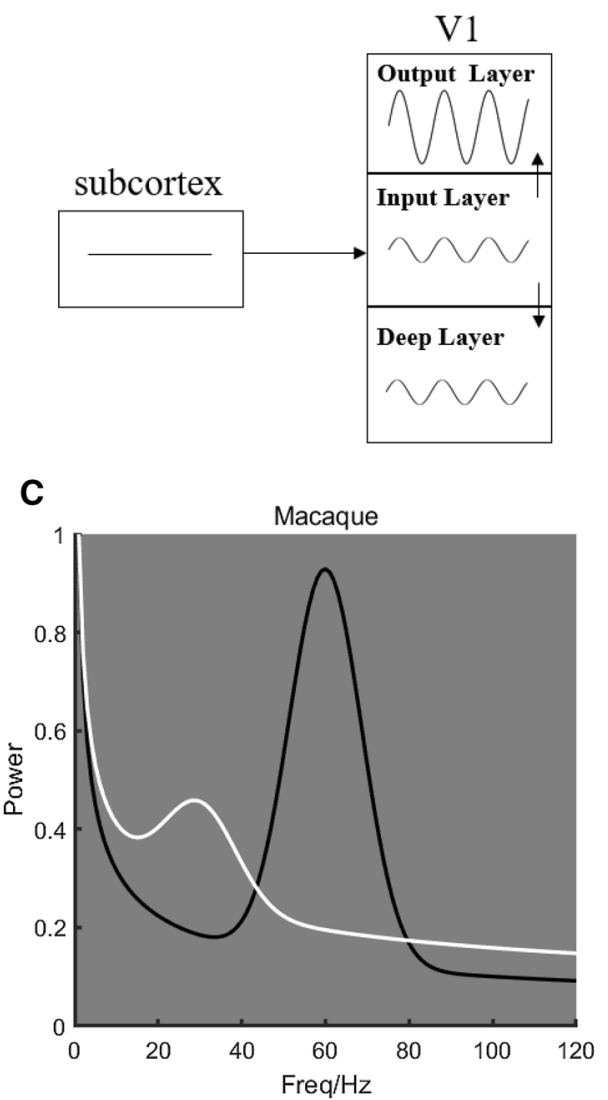

B

Mouse

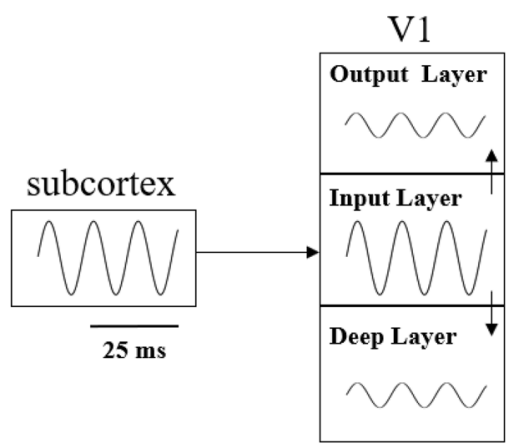

D

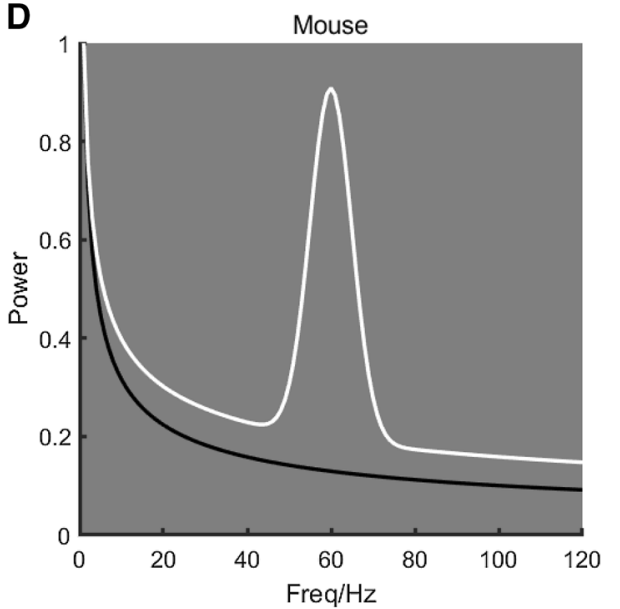

Computational models with interaction of excitation and inhibition (Fig. 6A) (Xing et al. 2012a; Chariker et al. 2016, 2018; Mejias et al. 2016) (Fig. 6DE) have made progress in understanding stochastic and stimulus-dependent properties of gamma. The gamma peaks are strongly influenced by different types of neural circuits. Local circuit models, have successfully achieved the stochastic properties observed in experiments (Xing et al. 2012a; Chariker et al. 2018). Furthermore, Jia and colleague (2013b) introduced feedback connections in the V1 model (Fig. 6B) and explained many observations concerning the changes of gamma power and frequency with different stimuli, including stimulus contrast, size, orientation, and noise-masking stimuli (Jia et al. 2013b) (Fig. 6F). More recently, Han et al. (2021) introduced horizontal connections (HC) into a large-scale V1 model (Fig. 6C). They found that $\mathrm{HC}$ could generate a new gamma around $30 \mathrm{~Hz}$ (slow gamma) which is different from fast gamma (around 50-60 Hz)(Fig. 6G). The two gamma band peaks that emerge in the Han et al. (2021) model are highly consistent with experimental findings for two distinct gammas in macaque V1(Fig. 4D).

Up to now, computational models have provided possible mechanisms for various gamma band phenomena in visual cortex, but there are still two main shortcomings. The first is that the predicted effects on gamma rhythms from feedback and horizontal neural circuits in the model should be further confirmed by experiments. Another one is that most of the models to explain stimulus dependence and stochastic property are at the mean-field level. Spiking neuron models (like Chariker et al. 2018) are closer to the actual response of neurons and will play a greater role in the future.

\section{Concluding Remarks}

Although the cognitive functions of gamma band peaks in cortical population activity are still unclear, it is apparent that gamma is related to multiple neural circuits, suggested by computational studies. As an essential feature for a complex dynamic system (the brain), gamma band peaks are crucial experimental data that should help us to understand neural circuits underlying brain functions. From both experimental and computational sides, more work on gamma band activity is needed for understanding its role in normal cognitive functions and abnormal mental states of the brain. 
A

B

Local E-I

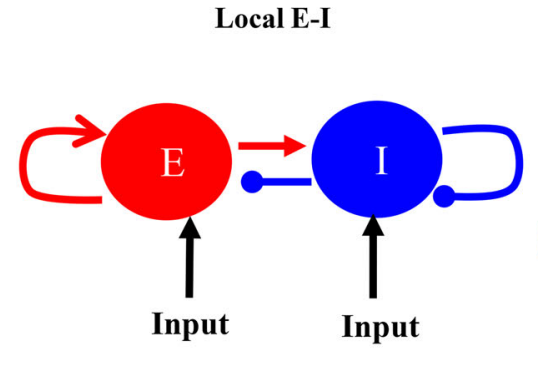

D

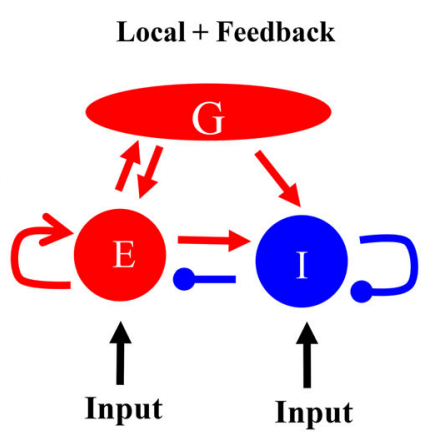

C Local + Feedback + Horizontal
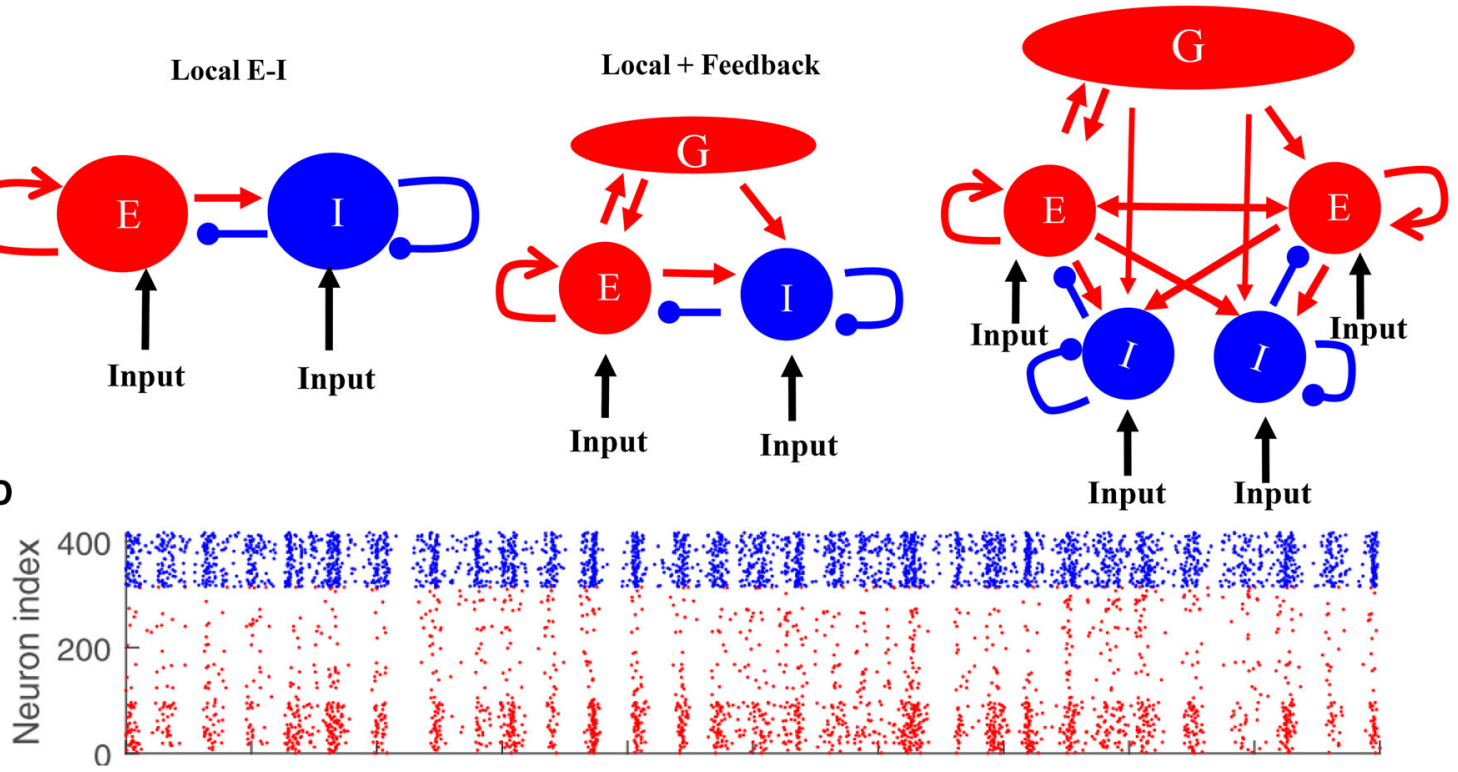

E

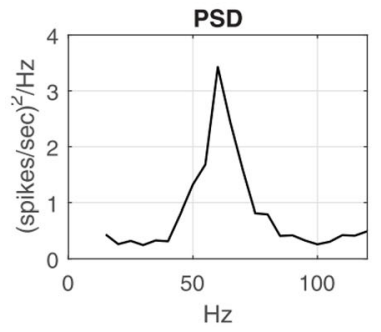

$\mathbf{F}$

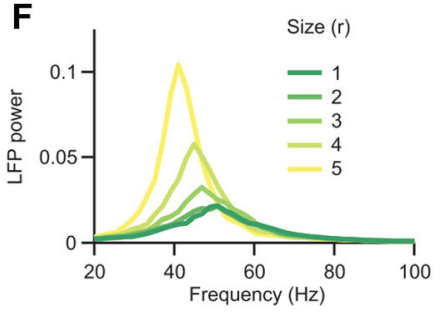

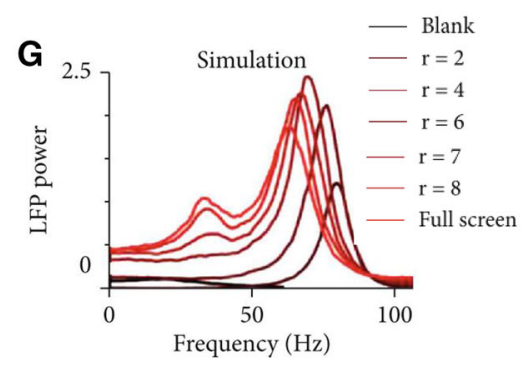

Fig. 6 Architectures and connectivity patterns of models for generating narrow-band gamma rhythms. A shows the PING model's basic framework (mutually connected pyramidal cell and interneuron). B shows a model based on the PING model in A, but it has an additional feedback component, which is driven by the E neurons and provided excitatory feedback to both E and I (Jia et al. 2013a, b). $\mathbf{C}$ shows a model based on the model in $\mathbf{B}$, with added horizontal connections (HC) in V1 (Han et al. 2021). D (replotted based on Chariker et al. 2018) shows the raster of spikes generated in a large

Acknowledgements This work was supported by the National Natural Science Foundation of China Grant 32171033 (DX), the Fundamental Research Funds for the Central Universities (DX), the 111 Project Grant (BP0719032) (DX), the Open Research Fund of the State Key Laboratory of Cognitive Neuroscience and Learning (DX), and by grant 1734854 from the US National Science Foundation (RS).

Author contributions All authors wrote the paper.

\section{Declarations}

Conflict of interest The authors declare no competing interest.

Open Access This article is licensed under a Creative Commons Attribution 4.0 International License, which permits use, sharing, adaptation, distribution and reproduction in any medium or format, as long as you give appropriate credit to the original author(s) and the source, provide a link to the Creative Commons licence, and indicate if changes were made. The images or other third party material in this scale spiking neural network (in a patch of $400 \mathrm{E}$ - and I- neurons in a cortical hyper-column). The spiking neuron model is also able to generate gamma band peaks with stochastic inputs, which is shown in the Power Spectral Density (PSD) plot in $\mathbf{E}$. F is replotted based on Jia et al. (2013a, b). It shows the simulated power spectra of LFP for gratings of different sizes based on the model shown in $\mathbf{B}$. $\mathbf{G}$ is replotted based on Han et al. (2021). It shows power spectra of the LFP induced by stimuli at different sizes in the model shown in $\mathbf{C}$

article are included in the article's Creative Commons licence, unless indicated otherwise in a credit line to the material. If material is not included in the article's Creative Commons licence and your intended use is not permitted by statutory regulation or exceeds the permitted use, you will need to obtain permission directly from the copyright holder. To view a copy of this licence, visit http://creativecommons. org/licenses/by/4.0/.

\section{References}

Adrian ED (1942) Olfactory reactions in the brain of the hedgehog. J Physiol 100:459-473

Adrian ED (1950) The electrical activity of the mammalian olfactory bulb. Electroencephalogr Clin Neurophysiol 2(1):377-388

Akam TE, Kullmann DM (2012) Efficient "communication through coherence" requires oscillations structured to minimize interference between signals. Plos Comput Biol 8(11):e1002760. https:// doi.org/10.1371/journal.pcbi.1002760 
Bartolo MJ, Gieselmann MA, Vuksanovic V et al (2011) Stimulusinduced dissociation of neuronal firing rates and local field potential gamma power and its relationship to the resonance blood oxygen level-dependent signal in macaque primary visual cortex. Eur J Neurosci 34:1857-1870

Bastos AM, Briggs F, Alitto HJ et al (2014) Simultaneous recordings from the primary visual cortex and lateral geniculate nucleus reveal rhythmic interactions and a cortical source for gammaband oscillations. J Neurosci 34:7639-7644

Bastos AM, Loonis R, Kornblith S et al (2018) Laminar recordings in frontal cortex suggest distinct layers for maintenance and control of working memory. Proc Natl Acad Sci U S A 115:1117-1122

Bauer R, Brosch M, Eckhorn R (1995) Different rules of spatial summation from beyond the receptive field for spike rates and oscillation amplitudes in cat visual cortex. Brain Res 669:291-297

Bauer EP, Paz R, Paré D (2007) Gamma oscillations coordinate amygdalo-rhinal interactions during learning. J Neurosci 27:9369-9379

Belluscio MA, Mizuseki K, Schmidt R et al (2012) Cross-frequency phase-phase coupling between theta and gamma oscillations in the hippocampus. J Neurosci 32:423-435

Benchenane K, Tiesinga PH, Battaglia FP (2011) Oscillations in the prefrontal cortex: a gateway to memory and attention. Curr. Opin Neurobiol 21(3):475-485

Berens P, Keliris GA, Ecker AS et al (2008) Comparing the feature selectivity of the gamma-band of the local fi eld potential and the underlying spiking activity in primate visual cortex. Front Syst Neurosci 2:1-11

Bharmauria V, Bachatene L, Ouelhazi A et al (2016) Interplay of orientation selectivity and the power of low- and high-gamma bands in the cat primary visual cortex. Neurosci Lett 620:14-19. https://doi.org/10.1016/j.neulet.2016.03.033

Börgers C (2017) An introduction to modeling neuronal dynamics. Springer, Cham, pp 255-267

Borgers C, Kopell NJ (2008) Gamma oscillations and stimulus selection. Neural Comput 20:383-414. https://doi.org/10.1162/ neco.2007.07-06-289

Bouyer JJ, Montaron MF, Rougeul A (1981) Fast fronto-parietal rhythms during combined focused attentive behaviour and immobility in cat: cortical and thalamic localizations. Electroencephalogr Clin Neurophysiol 51(3):244-252

Bragin A, Jandó G, Nádasdy Z et al (1995) Gamma (40-100 Hz) oscillation in the hippocampus of the behaving rat. J Neurosci $15: 47-60$

Burns SP, Xing D, Shelley MJ, Shapley RM (2010) Searching for autocoherence in the cortical network with a time-frequency analysis of the local field potential. J Neurosci 30:4033-4047

Burns SP, Xing D, Shapley RM (2011) Is gamma-band activity in the local field potential of v1 cortex a "clock" or filtered noise? J Neurosci 31:9658-9664

Buzsáki G (2009) Rhythms of the brain. Oxford univerisity Press, Oxford

Buzśaki G, Wang XJ (2012) Mechanisms of gamma oscillations. Annu Rev Neurosci 35:203-225

Canolty RT, Edwards E, Dalal SS et al (2006) High gamma power is phase-locked to theta oscillations in human neocortex. Science 313:1626-1628. https://doi.org/10.1126/science.1128115

Cardin JA, Palmer LA, Contreras D (2005) Stimulus-dependent $\gamma$ $(30-50 \mathrm{~Hz})$ oscillations in simple and complex fast rhythmic bursting cells in primary visual cortex. J Neurosci 25:5339-5350

Cardin JA, Carlén M, Meletis K et al (2009) Driving fast-spiking cells induces gamma rhythm and controls sensory responses. Nature 459:663-667

Castelo-Branco M, Neuenschwander S, Singer W (1998) Synchronization of visual responses between the cortex, lateral geniculate nucleus, and retina in the anesthetized cat. J Neurosci 18:6395-6410

Chariker L, Shapley R, Young LS (2016) Orientation selectivity from very sparse LGN inputs in a comprehensive model of macaque V1 cortex. J Neurosci 36:12368-12384

Chariker L, Shapley R, Young LS (2018) Rhythm and synchrony in a cortical network model. J Neurosci 38:8621-8634

Chrobak JJ, Buzsáki G (1998) Gamma oscillations in the entorhinal cortex of the freely behaving rat. J Neurosci. https://doi.org/10. 1523/jneurosci.18-01-00388.1998

Colgin LL (2011) Oscillations and hippocampal-prefrontal synchrony. Curr Opin Neurobiol 21(3):467-474

Colgin LL, Denninger T, Fyhn M et al (2009) Frequency of gamma oscillations routes flow of information in the hippocampus. Nature 462:353-357

Draguhn A, Buzsáki G (2004) Neuronal oscillations in cortical networks. Science 304:1926-1930

Eckhorn R, Bauer R, Jordan W et al (1988) Coherent oscillations: a mechanism of feature linking in the visual cortex? - Multiple electrode and correlation analyses in the cat. Biol Cybern 60(2):121-130

Edden RAE, Muthukumaraswamy SD, Freeman TCA, Singh KD (2009) Orientation discrimination performance is predicted by GABA concentration and gamma oscillation frequency in human primary visual cortex. J Neurosci 29:15721-15726. https://doi. org/10.1523/JNEUROSCI.4426-09.2009

Engel AK, König P, Kreiter AK et al (1992) Temporal coding in the visual cortex: new vistas on integration in the nervous system. Trends Neurosci 15(6):218-226

Feingold J, Gibson DJ, Depasquale B, Graybiel AM (2015) Bursts of beta oscillation differentiate postperformance activity in the striatum and motor cortex of monkeys performing movement tasks. Proc Natl Acad Sci U S A 112:13687-13692

Fernández-Ruiz A, Oliva A, Soula M et al (2021) Gamma rhythm communication between entorhinal cortex and dentate gyrus neuronal assemblies. Science. https://doi.org/10.1126/science. abf3119

Friedman-Hill S, Maldonado PE, Gray CM (2000) Dynamics of striate cortical activity in the alert macaque: I. Incidence and stimulus-dependence of gamma-band neuronal oscillations. Cereb Cortex 10(11):1105-1116

Frien A, Eckhorn R, Bauer R et al (1994) Stimulus-specific fast oscillations at zero phase between visual areas V1 and V2 of awake monkey. Neuroreport 5(17):2273-2277

Fries P (2005) A mechanism for cognitive dynamics: neuronal communication through neuronal coherence. Trends Cogn Sci 9:474-480

Fries P (2009) Neuronal gamma-band synchronization as a fundamental process in cortical computation. Annu Rev Neurosci 32:209-224

Fries P (2015) Rhythms for cognition: communication through coherence. Neuron 88:220-235

Fries P, Reynolds JH, Rorie AE, Desimone R (2001) Modulation of oscillatory neuronal synchronization by selective visual attention. Science 291:1560-1563

Fries P, Nikolić D, Singer W (2007) The gamma cycle. Trends Neurosci 30(7):309-316

Fujioka T, Trainor LJ, Large EW, Ross B (2009) Beta and gamma rhythms in human auditory cortex during musical beat processing. Annal N Y Acad Sci 1169(1):89-92

Gail A, Brinksmeyer HJ, Eckhorn R (2000) Contour decouples gamma activity across texture representation in monkey striate cortex. Cereb Cortex 10:840-850

Galuske RAW, Munk MHJ, Singer W (2019) Relation between gamma oscillations and neuronal plasticity in the visual cortex. 
Proc Natl Acad Sci U S A 116:23317-23325. https://doi.org/10. 1073/pnas. 1901277116

Gielen S, Krupa M, Zeitler M (2010) Gamma oscillations as a mechanism for selective information transmission. Biol Cybern 103:151-165

Gieselmann MA, Thiele A (2008) Comparison of spatial integration and surround suppression characteristics in spiking activity and the local field potential in macaque V1. Eur $\mathbf{J}$ Neurosci 28:447-459

Gonzalez-Burgos G, Lewis DA (2012) NMDA receptor hypofunction, parvalbumin-positive neurons, and cortical gamma oscillations in schizophrenia. Schizophr Bull. https://doi.org/10.1093/schbul/ sbs010

Grabowska MJ, Jeans R, Steeves J, van Swinderen B (2020) Oscillations in the central brain of Drosophila are phase locked to attended visual features. Proc Natl Acad Sci U S A 117:29925-29936. https://doi.org/10.1073/pnas.2010749117

Gray CM, Di Prisco GV (1997) Stimulus-dependent neuronal oscillations and local synchronization in striate cortex of the alert cat. J Neurosci 17(9):3239-3253

Gray CM, McCormick DA (1996) Chattering cells: Superficial pyramidal neurons contributing to the generation of synchronous oscillations in the visual cortex. Science. https://doi.org/10.1126/ science.274.5284.109

Gray CM, Singer W (1989a) Stimulus-specific neuronal oscillations in orientation columns of cat visual cortex. Proc Natl Acad Sci U S A 86:1698-1702

Gray CM, Singer W (1989) Stimulus-specific neuronal oscillations in orientation columns of cat visual cortex. Proc Natl Acad Sci U S A 86(5):1698-1702

Gray CM, König P, Engel AK, Singer W (1989) Oscillatory responses in cat visual cortex exhibit inter-columnar synchronization which reflects global stimulus properties. Nature. https://doi. org $/ 10.1038 / 338334 \mathrm{a} 0$

Gregoriou GG, Gotts SJ, Zhou H, Desimone R (2009) Highfrequency, long-range coupling between prefrontal and visual cortex during attention. Science 324(5931):1207-1210

Gross J, Hoogenboom N, Thut G et al (2013) Speech rhythms and multiplexed oscillatory sensory coding in the human brain. PLoS Biol 11(12):e1001752

Grossberg S (1976) Adaptive pattern classification and universal recoding: II. Feedback, expectation, olfaction, illusions. Biol Cybern 23(4): 187-202

Han C, Wang B, Yang G et al (2020) Neural mechanism of orientation selectivity for distinct gamma oscillations in cat V1. J vis $20: 1116$

Han C, Wang T, Wu Y et al (2021) The generation and modulation of distinct gamma oscillations with local, horizontal, and feedback connections in the primary visual cortex: a model study on largescale networks. Neural Plast 2021:8874516

Hawellek DJ, Wong YT, Pesaran B (2016) Temporal coding of reward-guided choice in the posterior parietal cortex. Proc Natl Acad Sci U S A 113(47):13492-13497

Henrie JA, Shapley R (2005) LFP power spectra in V1 cortex: the graded effect of stimulus contrast. J Neurophysiol 94:479-490

Hermes D, Miller KJ, Wandell BA, Winawer J (2015) Stimulus dependence of gamma oscillations in human visual cortex. Cereb Cortex 25:2951-2959

Jensen O, Kaiser J, Lachaux JP (2007) Human gamma-frequency oscillations associated with attention and memory. Trends Neurosci 30(7):317-324

Jia X, Kohn A (2011) Gamma rhythms in the brain. PLoS Biol 9:2-5

Jia X, Smith MA, Kohn A (2011) Stimulus selectivity and spatial coherence of gamma components of the local field potential. J Neurosci 31:9390-9403
Jia X, Tanabe S, Kohn A (2013a) Gamma and the coordination of spiking activity in early visual cortex. Neuron 77:762-774

Jia X, Xing D, Kohn A (2013b) No consistent relationship between gamma power and peak frequency in macaque primary visual cortex. J Neurosci 33:17-25

Kayser C, Ko P (2004) Short communication stimulus locking and feature selectivity prevail in complementary frequency ranges of V1 local ${ }^{\circledR}$ eld potentials. Neuroscience 19:485-489

Kayser C, Salazar RF, König P (2003) Responses to natural scenes in cat VI. J Neurophysiol 90(3):1910-1920

Kim H, Ährlund-Richter S, Wang X et al (2016) Prefrontal parvalbumin neurons in control of attention. Cell 164(1-2):208-218

Kirschfeld K (1992) Oscillations in the insect brain: do they correspond to the cortical $\gamma$ - waves of vertebrates? Proc Natl Acad Sci U S A 89:4764-4768. https://doi.org/10.1073/pnas.89. 10.4764

Kreiter AK, Singer W (1996) Stimulus-dependent synchronization of neuronal responses in the visual cortex of the awake macaque monkey. J Neurosci 16(7):2381-2396

Kucewicz MT, Berry BM, Kremen V et al (2017) Dissecting gamma frequency activity during human memory processing. Brain 140:1337-1350

Lakatos P, Shah AS, Knuth KH et al (2005) An oscillatory hierarchy controlling neuronal excitability and stimulus processing in the auditory cortex. J Neurophysiol 94(3):1904-1911

Lewis DA, Curley AA, Glausier JR, Volk DW (2012) Cortical parvalbumin interneurons and cognitive dysfunction in schizophrenia. Trends Neurosci 35(1):57-67

Lima B, Singer W, Chen NH, Neuenschwander S (2010) Synchronization dynamics in response to plaid stimuli in monkey V1. Cereb Cortex 20:1556-1573

Maldonado PE, Friedman-Hill S, Gray CM (2000) Dynamics of striate cortical activity in the alert macaque: II. Fast time scale synchronization. Cereb Cortex 10(11):1105-1116

Mejias JF, Murray JD, Kennedy H, Wang XJ (2016) Feedforward and feedback frequency-dependent interactions in a large-scale laminar network of the primate cortex. Sci Adv. https://doi.org/ 10.1126/sciadv. 1601335

Michalareas G, Vezoli J, van Pelt S et al (2016) Alpha-beta and gamma rhythms subserve feedback and feedforward influences among human visual cortical areas. Neuron 89:384-397. https:// doi.org/10.1016/j.neuron.2015.12.018

Milner PM (1974) A model for visual shape recognition. Psychol Rev 81(6):521-535

Murty DVPS, Shirhatti V, Ravishankar P, Ray S (2018) Large visual stimuli induce two distinct gamma oscillations in primate visual cortex. J Neurosci 38:2730-2744

Neuenschwander S, Singer W (1996) Long-range synchronization of oscillatory light responses in the cat retina and lateral geniculate nucleus. Nature 379(6567):728-733

Neville KR, Haberly LB (2003) Beta and gamma oscillations in the olfactory system of the urethane-anesthetized rat. J Neurophysiol 90(6):3921-3930

Oke OO, Magony A, Anver H et al (2010) High-frequency gamma oscillations coexist with low-frequency gamma oscillations in the rat visual cortex in vitro. Eur J Neurosci 31:1435-1445

Onorato I, Neuenschwander S, Hoy J et al (2020) A distinct class of bursting neurons with strong gamma synchronization and stimulus selectivity in monkey V1. Neuron 105:180-197.e5. https://doi.org/10.1016/j.neuron.2019.09.039

Pesaran B, Pezaris JS, Sahani M et al (2002) Temporal structure in neuronal activity during working memory in macaque parietal cortex. Nat Neurosci 5(8):805-811 
Peter A, Uran C, Klon-Lipok J et al (2019) Surface color and predictability determine contextual modulation of V1 firing and gamma oscillations. Elife. https://doi.org/10.7554/eLife.42101

Quilichini P, Sirota A, Buzsáki G (2010) Intrinsic circuit organization and theta-gamma oscillation dynamics in the entorhinal cortex of the rat. J Neurosci. https://doi.org/10.1523/JNEUROSCI.132710.2010

Ray S, Maunsell JHR (2010) Differences in gamma frequencies across visual cortex restrict their possible use in computation. Neuron 67:885-896

Ray S, Maunsell JHR (2011) Different origins of gamma rhythm and high-gamma activity in macaque visual cortex. PLoS Biol 9(4):e1000610

Roberts MJ, Lowet E, Brunet NM et al (2013) Robust gamma coherence between macaque V1 and V2 by dynamic frequency matching. Neuron 78:523-536

Roelfsema PR, Lamme VAF, Spekreijse H (2004) Synchrony and covariation of firing rates in the primary visual cortex during contour grouping. Nat Neurosci 7:982-991

Saleem AB, Lien AD, Krumin M et al (2017) Subcortical source and modulation of the narrowband gamma oscillation in mouse visual cortex. Neuron 93:315-322

Samonds JM, Bonds AB (2005) Gamma oscillation maintains stimulus structure-dependent synchronization in cat visual cortex. J Neurophysiol 93:223-236. https://doi.org/10.1152/jn. 00548.2004

Saraf S, Young LS (2021) Malleability of gamma rhythms enhances population-level correlations. J Comput Neurosci 49:189-205

Schneider M, Broggini AC, Dann B et al (2021) A mechanism for inter-areal coherence through communication based on connectivity and oscillatory power. Neuron. https://doi.org/10.1016/j. neuron.2021.09.037

Schoffelen JM, Oostenveld R, Fries P (2005) Neuronal coherence as a mechanism of effective corticospinal interaction. Science 308:111-113

Shadlen MN, Movshon JA (1999) Synchrony unbound: a critical evaluation of the temporal binding hypothesis. Neuron 24:67-77

Shirhatti V, Ray S (2018) Long-wavelength (reddish) hues induce unusually large gamma oscillations in the primate primary visual cortex. Proc Natl Acad Sci U S A 115:4489-4494

Siegel M, König P (2003) A functional gamma-band defined by stimulus-dependent synchronization in area 18 of awake behaving cats. J Neurosci 23:4251-4260

Singer W (1999) Neuronal synchrony: a versatile code for the definition of relations? Neuron 24(1):49-65

Singer W (1999) Striving for coherence. Nature 397(6718):391-393

Singer W (2009) Distributed processing and temporal codes in neuronal networks. Cogn Neurodyn 3(3):189-196

Singer W (2018) Neuronal oscillations: unavoidable and useful? Eur J Neurosci 48(7):2389-2398

Singer W (2021) Recurrent dynamics in the cerebral cortex: integration of sensory evidence with stored knowledge. Proc Natl Acad Sci U S A 118:1-12

Singer W, Gray CM (1995) Visual feature integration and the temporal correlation hypothesis. Annu Rev Neurosci 18:555-586

Spaak E, Bonnefond M, Maier A et al (2012) Layer-specific entrainment of gamma-band neural activity by the alpha rhythm in monkey visual cortex. Curr Biol 22:2313-2318. https://doi. org/10.1016/j.cub.2012.10.020

Storchi R, Bedford RA, Martial FP et al (2017) Modulation of fast narrowband oscillations in the mouse retina and dLGN according to background light intensity. Neuron 93:299-307

Subhash Chandran KS, Seelamantula CS, Ray S (2018) Duration analysis using matching pursuit algorithm reveals longer bouts of gamma rhythm. J Neurophysiol 119:808-821. https://doi.org/10. 1152/jn.00154.2017
Swettenham JB, Muthukumaraswamy SD, Singh KD (2013) BOLD responses in human primary visual cortex are insensitive to substantial changes in neural activity. Front Hum Neurosci 7:1-11. https://doi.org/10.3389/fnhum.2013.00076

Tallon-Baudry C, Bertrand O (1999) Oscillatory gamma activity in humans and its role in object representation. Trends Cogn Sci 3:151-162. https://doi.org/10.1016/S1364-6613(99)01299-1

Thiele A, Stoner G (2003) Neuronal synchrony does not correlate with motion coherence in cortical area MT. Nature 421:366-370

Uhlhaas PJ, Singer W (2006) Neural synchrony in brain disorders: relevance for cognitive dysfunctions and pathophysiology. Neuron 52(1):155-168

Uhlhaas PJ, Singer W (2010) Abnormal neural oscillations and synchrony in schizophrenia. Nat Rev Neurosci 11(2):100-113

Uhlhaas PJ, Singer W (2012) Neuronal dynamics and neuropsychiatric disorders: toward a translational paradigm for dysfunctional large-scale networks. Neuron 75:963-980

Van Kerkoerle T, Self MW, Dagnino B et al (2014) Alpha and gamma oscillations characterize feedback and feedforward processing in monkey visual cortex. Proc Natl Acad Sci U S A 111:14332-14341

Veit J, Hakim R, Jadi MP et al (2017) Cortical gamma band synchronization through somatostatin interneurons. Nat Neurosci 20:951-959

Vianney-Rodrigues P, Iancu OD, Welsh JP (2011) Gamma oscillations in the auditory cortex of awake rats. Eur J Neurosci 33(1):119-129

Vinck M, Womelsdorf T, Buffalo EA et al (2013) Attentional modulation of cell-class-specific gamma-band synchronization in awake monkey area V4. Neuron 80:1077-1089

von der Malsburg C (1994) The correlation theory of brain function. Springer, New York

Wang XJ (2010) Neurophysiological and computational principles of cortical rhythms in cognition. Physiol Rev 90:1195-1268

Wang XJ, Buzsáki G (1996) Gamma oscillation by synaptic inhibition in a hippocampal interneuronal network model. J Neurosci 16:6402-6413

Wang B, Han C, Wang T et al (2021) Superimposed gratings induce diverse response patterns of gamma oscillations in primary visual cortex. Sci Rep 11(1):1-20

Welle CG, Contreras D (2017) New light on gamma oscillations. Neuron 93:247-249. https://doi.org/10.1016/j.neuron.2017.01. 003

Womelsdorf T, Fries P (2007) The role of neuronal synchronization in selective attention. Curr Opin Neurobiol 17(2):154-160

Womelsdorf T, Schoffelen JM, Oostenveld R et al (2007) Modulation of neuronal interactions through neuronal synchronization. Science 316:1609-1612

Womelsdorf T, Lima B, Vinck M et al (2012) Orientation selectivity and noise correlation in awake monkey area V1 are modulated by the gamma cycle. Proc Natl Acad Sci U S A 109:4302-4307

Xing D, Yeh CI, Shapley RM (2010) Generation of black-dominant responses in V1 cortex. J Neurosci 30:13504-13512

Xing D, Shen Y, Burns S et al (2012a) Stochastic generation of gamma-band activity in primary visual cortex of awake and anesthetized monkeys. J Neurosci 32:13873-13880

Xing D, Yeh CI, Burns S, Shapley RM (2012b) Laminar analysis of visually evoked activity in the primary visual cortex. Proc Natl Acad Sci U S A 109:13871-13876

Xing D, Yeh CI, Gordon J, Shapley RM (2014) Cortical brightness adaptation when darkness and brightness produce different dynamical states in the visual cortex. Proc Natl Acad Sci U S A 111:1210-1215

Yuval-Greenberg S, Tomer O, Keren AS et al (2008) Transient induced gamma-band response in EEG as a manifestation of miniature saccades. Neuron 58:429-441 
Zhou Z, Bernard MR, Bonds AB (2008) Deconstruction of spatial integrity in visual stimulus detected by modulation of synchronized activity in cat visual cortex. J Neurosci 28(14):3759-3768
Publisher's Note Springer Nature remains neutral with regard to jurisdictional claims in published maps and institutional affiliations. 